\title{
Use and Efficasy of Different Imaging Techniques in Flank Pain
}

\author{
Dr. Sanjeev K Gupta \\ Assoc. Prof. Deptt. Surgery I G Medical College, Shimla.
}

\begin{abstract}
Acute flank pain is a common presentation in patients attending casualty/OPD and is representation of many diseases. Urolithiasis is appears to be the most frequent cause of frequent cause of flank pain, affecting $3 \%$ to $5 \%$ of the population in urbanized countries ${ }^{I}$. Urolithiasis is estimated to have a lifetime incidence of 5 12 percent usually presenting in patients 30-60 years of age and is approximately three times more common in males, ${ }^{1,2}$ and out of these has recurrence rate approximate $50 \%{ }^{3}$ It is; therefore, important to address this problem after making early diagnosis by using traditional different imaging techniques like X-rays (X-Ray KUB) and conventional ultrasound (USG)), intravenous pyelogram (IVP) and Non-Contrast CT (NCCT) which is becoming increasingly popular due to its high accuracy, less time consuming, no contrast allergy and detecting radiolucent stones in addition to alternate diagnosis .
\end{abstract}

Keywords: Flank pain; Alternate diagnosis; Imaging techniques; Non Contrast CT; Radiation Exposure;

\section{Introduction}

Among the presently available modalities, X-RAY KUB alone has low sensitivity \& specificity due to bowel gas, missing out radiolucent stones and inability to differentiate stones from phlebolith calcified lymph nodes and other calcified structures though its ability to detect location \& size site helps in preoperative planning. Ultrasonography (USG) is the modality of choice in pregnant patients and children to avoid radiation. As a bedside procedure, it is quickly performed to see secondary signs of presence of stone ${ }^{5}$. As stated earlier, ultrasound is less sensitive at detecting stones in the ureter than in the kidney and its limitation in identifying stones location, size especially in obese.

Although IVP has remained traditional "gold standard" in the evaluation of renal pain for decades, its use has now fallen out of favour with new imaging technique NCCT owing not only its high accuracy ${ }^{6-8}$ of detection of stone including translucent stone associated secondary changes in excretory system but also detecting alternate diagnosis including life threatening conditions. In this study IVP is not used in order to avoid reaction of contrast, its nephrotoxicity especially in already obstructed excretory system due to stone in deranged kidney function.

This study is an attempt to analyse the efficacy and safety of individual imaging modality in management of urolithiasis as well as alternate diseases when present.

\section{Methods}

In this study, 50 patients above 14 yrs of both sexes presenting first time with flank pain with or without other urinary symptoms except pregnancy were included in Department of Surgery, IGMC Shimla. In each case detail history followed by clinical examination and further investigations were carried out to know the exact cause of flank pain and its management thereof.

In all cases x-ray KUB, ultrasound followed by NCCT of whole of abdominal was done. In these patients, in addition, secondary changes due to urolithiasis were also seen with USG \& NCCT. Incidental pathologies/diagnosis causing flank pain were seen \& managed accordingly. Traditional imaging technique, IVP which was not without risk of dye reaction \& nephrotoxicity in already obstructive kidney (in late arrival of patients in this hilly terrain) therefore not used. Efficacies of individual imaging technique and in combination were evaluated.

\section{Results}

The following observations were made as majority of patients were between 20 to 60 years of age $(84 \%)$. The female to male ratio was 1.2:1. The most common presenting complaint was flank pain in 49 patients (98\%) followed by increase in frequency of urine in 34 patients (68\%), burning maturation present in 26 patients (52\%), Hematuria present in 6 patients (12\%) and fever was present in 15 patients (30\%). The most common biochemical abnormality was raised serum creatinine, which was raised in 18 patients (36\%). Blood urea was elevated in 9 patients $(18 \%)$.

In this study, out of 50 patients, 39 were diagnosed with urolithiasis whereas 11 were without any urolithiasis. Out of these 11 cases, 5 cases had UTI, 2 cases were having chronic cholecystitis, and one case was of mesenteric lymphadenitis \& others one each of leucorrhea one recently passed stone and one diagnosed as 
rhabdomyolysis. Incidental diagnosis were observed in this study by USG were in 9 cases while with NCCT it was in 13 cases.

X-Ray KUB alone, in the present study, detected stones in only 14 cases out of 39 i.e. sensitivity $35.89 \%$ and specificity $72.72 \%$ whereas USG detected urolithiasis in 20 cases out of actual 39 cases i.e. sensitivity $51.28 \%$ and specificity $90.90 \%$. When combined KUB-USG detected stones in 35 out of 39 patients with sensitivity $89.74 \%$ and specificity $90.90 \%$. NCCT-KUB correctly diagnosed urolithiasis in all the 39 out of 50 patients irrespective of their locations and size i.e. 15 in kidney, 14 in ureter, 8 cases both in kidney and ureter and one each case of urethra and bladder. It also detected stones of size ranged from $2 \mathrm{~mm}$ to $44 \mathrm{~mm}$ thereby sensitivity of $100 \%$. It also correctly diagnosed absence of urolithiasis in 10 out of 11 negative cases i.e. specificity of $90.90 \%$ in the present study.

Table-1: Detection of Urolithiasis by Kub-Usg Vs. Ncct-Kub

\begin{tabular}{|c|c|c|c|c|c|c|c|c|c|}
\hline \multicolumn{2}{|c|}{ Urolithiasis } & \multicolumn{2}{|c|}{$\begin{array}{l}\text { Intervention/surge } \\
\text { ry }\end{array}$} & & \multicolumn{2}{|c|}{ Urolithiasis } & \multicolumn{2}{|c|}{ Intervention/surgery } & \multirow[b]{2}{*}{ Total } \\
\hline & & Present & Absent & Total & & & Present & Absent & \\
\hline \multirow{3}{*}{ 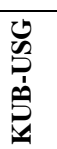 } & Present & 35 & 1 & 36 & \multirow{3}{*}{$\underbrace{\tilde{u}}_{\mathbf{Z}}$} & Present & 39 & 1 & 40 \\
\hline & Absent & 4 & 10 & 14 & & Absent & $\mathbf{0}$ & 10 & 10 \\
\hline & Total & 39 & 11 & $\mathbf{5 0}$ & & Total & 39 & 11 & 50 \\
\hline
\end{tabular}

Small \& single non obstructive stones $(<8.00 \mathrm{~mm})$ seen on NCCT in 19 patients were put on a trial of Medical expulsive therapy. These patients were saved for undergoing any invasive intervention due to accurate detection of stone size. In total, 28 operative procedures were performed on 24 patients, however, 3 patients requiring more than one operative procedure. Ultrasound is also very good at identifying secondary signs of obstruction during renal colic. Besides urolithiasis, in the present study, ultrasonography was suggestive of hydronephrosis in $36(92 \%)$ and hydroureter in 24(61\%) of the studied 50 patients. The sensitivity and specificity of USG for hydronephrosis was $92.30 \%$ and $63.63 \%$ respectively. The sensitivity and specificity of USG for hydroureter in the present study was $61.54 \%$ and $81.82 \%$ respectively. This new imaging protocol of using NCCT-KUB in our institution, in concordance to literature studies, has demonstrated urolithiasis in 39 $(100 \%)$ out of the 39 patients diagnosed with urolithiasis per operatively. Out of the 39 cases 15 were of kidney stones, 14 were of ureteral stones, 8 cases were of both kidney and ureteral and 1 case each of urethral and bladder stone. The stone size diagnosed on NCCT ranged from $2 \mathrm{~mm}$ to $44 \mathrm{~mm})$. NCCT proved to have a sensitivity of $100 \%$ (39 out of 39 patients) in demonstration of urolithiasis and specificity of $90.90 \%$ (10 out of 11 patients).

\section{Discussions}

Radiological imaging techniques play a central role in management of patients presenting with suspected acute flank pain and early, accurate with precise size \& location of stone is of paramount importance for selection of intervention \& pre operative planning and also ruling out life threatening diseases like abdominal aortic aneurysm, renal mass, pyelonephritis, appendicitis ovarian cyst. Ultrasound has been shown to overestimate stone size which has direct implications in selecting the modalities of treatment. Moreover, in developing countries like India, patients present late with obstructive uropathy and deranged renal functions, use of traditional gold standards i.e. IVP is not without risk. Meanwhile, NCCT has become the referral standard in evaluation of suspected acute renal pain ${ }^{78}$ In as many as one third of patients acute flank pain can be due to other abdominal conditions and capability of NCCT to detect such conditions reliably make it modality of choice $^{9}$. Therefore, at our institutions new imaging protocol was introduced in July, 2013 replacing traditional approach of X-ray, USG or IVP with NCCT KUB. In order to evaluate the effectiveness of new approach with NCCT KUB, this study of 50 consecutive cases was undertaken.

In the present study, The sensitivity of X-ray KUB to detect urolithiasis in the present study was $35.89 \%$ and the specificity $72.72 \%$ similar to Levine and colleagues $(1997)^{4}$ reviewed 178 patients with acute flank pain finding KUBs with a sensitivity of $45 \%$ to $59 \%$ and specificity of $77 \%$ in detection of urinary tract calculi. When ultrasound is added to X-ray KUB is sensitivity and specificity is remarkably increased to $96 \%$, and $91 \%$ respectively as shown by Mitterberger et al $(2007)^{10}$ The use of tomography has been shown to improve the diagnostic accuracy of X-ray KUB in evaluation of urolithiasis by Goldwasser et al $\left(1989^{11}\right.$. In $46 \%$ of patient's additional stones were seen on tomograms versus KUB and in $8 \%$ of patients stones were not seen on KUB but identified on tomograms. The stone size was significantly smaller in the patients in whom ultrasound failed to diagnose a stone $(4 \mathrm{~mm}$ vs. $6 \mathrm{~mm})$. In addition to difficulties in identifying small stones, ultrasound has been on to overestimate stone size compared to NCCT. 
In the present study, USG correctly diagnosed urolithiasis in 20 out of total 39 patients with urolithiasis, with sensitivity, specificity, $51.28 \%, 90.90 \%$, respectively. The stone size diagnosed on USG ranged from $2 \mathrm{~mm}$ to $50 \mathrm{~mm}$. These results were comparable to a study by Fowler and colleagues $(2002)^{12}$. The advantage of no radiation exposure comes at a cost of decreased sensitivity and specificity, especially for ureteral calculi. Ultrasound is less accurate at imaging ureteral stones. In the present study, USG correctly diagnosed 10 ureteral stones $(45 \%)$ out of the total 22 ureteral stones seen eventually on NCCT, with sensitivity, specificity, of $45.45 \%, 63.63 \%$, respectively. This was similar to a study by Yilmaz and colleagues and also Sheafor et al $(2000)^{13}$, showing the sensitivity for ultlrasound diagnosing a ureteral stone $61 \%$ versus $96 \%$ for NCCT.

Ripolles et al (2004) ${ }^{14}$ observed similar results in a study, where ultrasound identified hydronephrosis in $95 \%$, ureteral dilation in $89 \%$ and perirenal fluid in $23 \%$ of the patients as in present study. Due to low individual sensitivities and specificities, the KUB should, therefore, always be paired with another imaging modality such ultrasound. In the present study X-ray KUB when combined with USG, correctly diagnosed urolithiasis in $35(70 \%)$ of the 39 patients with urolithiasis. It was recommended that plain X-ray KUB should be performed before the USG examination with the understanding that only USG confirms a positive urolithiasis since calcifications on abdominal films can be misleading.Ultrasound can be useful for follow up in patients who are diagnosed with distal ureteral stones with evidence for obstruction on NCCT and are offered conservative management to rule out persistent obstruction. It is also useful for follow up of patients who undergo uncomplicated surgical procedures to rule out new or residual obstruction. Additionally, in the absence of renal calculi or hydronephrosis, ultrasonography has additional role in diagnosis of alternate pathologies as reported by Patlas et al $(2001)^{9}$ comparing ultrasound and NCCT for the evaluation of renal colic in 62 patients, similar to present study.

The advantage of NCCT in diagnosing urolithiasis when stone has passed over IVP as observed by Ha M $(2004)^{15}$ in his study. Similarly, Varaneli MJ et al $(2001)^{16}$ commented on the secondary and indirect signs of calculus disease in the renal system detected on NCCT in the form of perinephric, stranding, ureteral dilatation, perinephric fluid, collecting system dilatation, periureteral stranding and nephromegaly. Secondary signs are indicative of a localized inflammatory reaction or irritation caused by the presence or passing of ureteral stones or other acute urinary obstruction. These indirect signs are thought to follow a well defined time course corresponding to the physiological changes caused by an acutely obstructing stone. The peak time of appearance of these secondary signs is reported to be 6 to 8 hours following obstruction, based on a study of 227 patients with stone diagnosis on $\mathrm{NCCT}^{16}$. There are other advantages NCCT holds over IVP and other imaging modalities for the evaluation of acute flank pain. NCCT is quickly performed and does not require intravenous contrast. Unlike plain radiography such as KUB and IVP, NCCT can detect stones of almost any composition. The exception to this, as studied by Sundaram CP et al $(1999)^{17}$ is stones formed by protease inhibitors such as indinavir, which may not be visible on NCCT. However, often in these cases, there are secondary signs of stones such as hydroureter and periureteral or perinephric inflammation which aide in the diagnosis ${ }^{17}$.

In present study, the false-positive result with NCCT is due to phleboliths to be differentiated from ureteral calculi as also pointed out by Boridy et al. Another limitation of false negative result has been reported, with rates ranging from $2 \%$ to $7 \%{ }^{18}$. These false negative results have been attributed to a probable combination of volume averaging (small stone size, i.e. $<2 \mathrm{~mm}$ relative to collimation) and stone composition. This is a particular problem seen in patients positive for human immunodeficiency virus who are being treated with the protease inhibitor indianvir. In these patients, the majority of calculi are low attenuating (matrix stones) on NCCT images ${ }^{18}$. It is, therefore, useful to look closely for secondary signs of obstruction, i.e. hydronephrosis and hydroureter.

There are, however, some concerns regarding the use of NCCT in suspected acute renal colic. Firstly, NCCT cannot access the functional status of kidneys, for which renal scan or IVP is additionally needed. Secondly, according to published reports, patients are usually exposed to a three to five times higher dose of radiation with NCCT compared to the dose with IVP $^{19}$. Hence, NCCT cannot be used in paediatric age group and in pregnant females. In addition, because patients with stone disease are often relatively young and may thus be repeat stone formers who may thus require multiple NCCT examinations during their life time, there should be an effort to reduce the radiation dose of $\mathrm{CT}^{20}$. The effective dose for a KUB and IVP has been reported to be approximately 1.3 and $3 \mathrm{mSv}$ respectively, by Mettler et al $(2008)^{21}$. The number of images obtained during an IVP affects the dose, with the more shots taken, the higher the effective dose. The effective dose of the KUB was calculated to be $0.67 \mathrm{mSv}$, and the effective dose of each tomogram was $1.1 \mathrm{mSv}$. The effective dose of a 'low-dose' NCCT was $3.04 \mathrm{mSv}$. Typically, when KUB and tomograms are performed, a KUB is taken along with 3-4 tomograms. This yields a total dose of 3.97-5.07 mSv. 
Use And Efficasy Of Different Imaging Techniques In Flank Pain

Table-2: Sampling of Relevant Literature of Urolithiasis and NCCT (Accuracy)

\begin{tabular}{|l|l|l|l|}
\hline \multicolumn{1}{|c|}{ Study } & Year & Sensitivity \% & Specificity \% \\
\hline Smith RC & 1996 & $95-98$ & $96-98$ \\
\hline Vieweg J et al & 1998 & $94-100$ & $92-100$ \\
\hline Jeong Ah Ryu & 2001 & 96 & 100 \\
\hline Tamm EP et al & 2003 & $94-100$ & $92-100$ \\
\hline S. Feroz et al & 2007 & 91 & 98 \\
\hline K. Patatas & 2012 & $97-98$ & $96-100$ \\
\hline Present study & 2016 & 100 & 90 \\
\hline
\end{tabular}

Also, with advancement in minimally invasive treatment options available for managing urolithiasis, like PCNL and ESWL, which results in small residual stone fragments in urinary tract, increasing number of NCCTs are being requested in follow up of such patients, thus increasing the radiation exposure per patient. And lastly, such a change in the use of NCCT can result in a significant impact on radiology workflow and budgets and can radically alter the diagnostic process. Although there are studies that have demonstrated a costequivalence between the two techniques, others are in agreement that NCCT introduces cost savings. This would thus make an even more compelling case for implementation of such an imaging protocol.

\section{Conclusion}

Accurate information regarding the presence, size and precise location of urolithiasis along with intra calyceal anatomy by the available imaging is the hallmark in deciding precise modality of management. But no single technique is ideal as associated with either adverse effect on body or havig low accuracy. The choice is also heavily dependent on local resources. NCCT-KUB in this study is the most accurate first line modality for precisely detecting urolithiasis. The present series of 50 consecutive cases highlighted the clinical utility of NCCT, due to its exceptional sensitivity and specificity for identifying a stone, size, composition, location or secondary changes, to only accurate diagnose it but also to diagnose alternate diseases/findings and thus help in determining appropriate surgical management. However, restriction of its use in paediatric patients and pregnant women due to the increased risks from radiation exposure, a low dose NCCT or a combination of X-ray KUB and USG can be used an alternative in these patients.

Indiscriminate use of NCCT not only increases the workload on radiology department but also risks the normal population especially females with flank pain due to UTI/ Leucorrhoea. USG/X-ray KUB should be used in follow up patients post operatively (ESWL/PCNL) with residual stone fragments rather than using NCCT.

\section{References}

[1]. Hiatt RA, Dales LG, Friedman GD, et al. Frequency of urolithiasis in a prepaid medical care program. Am J Epidemiol. 1982; 115:255-5

[2]. Kirpalani A, Khalili K, Lee S, et al. Renal colic: comparison of use and outcomes of unenhanced helical CT for emergency investigation in 1998 and 2002. Radiology. 2005; 236:554-8.

[3]. Sierakowski R, Finlayson B, Landes RR, et al. The frequency of urolithiasis in hospital discharge diagnoses in the United States. Invest Urol. 1978; 15:438-41.

[4]. Levine JA, Neitlich J, Verga M, et al. Ureteral calculi in patients with flank pain: correlation of plain radiography with unenhanced helical CT. Radiology. 1997; 204(1):27-31.

[5]. Fowler KA, Locken JA, Duchesne JH, et al. US for detecting renal calculi with nonenhanced CT as a reference standard. Radiology, 2002; 222:109-13.

[6]. Segal AJ, Spataro RF, Linke CA, et al. Diagnosis of nonopaque calculi by computed tomography. Radiology. 1978; 129:447-50.

[7]. Federle MP, Mc Aninch JW, Kaiser JA, et al. Computed tomography of urinary calculi. AJR Am J Roentgenol. 1981; 136:255-8.

[8]. Smith RC, Rosen field AT, Choe KA, et al. Acute flank pain: comparison of non-contrast-enhanced CT and intravenous urography. Radiology. 1995; 194:789-94.

[9]. Patlas M, Farkas A, Fisher D, et al. Ultrasound vs CT for the detection of ureteric stones in patients with renal colic. Br J Radiol. $2001 ; 74: 901-4$.

[10]. Mitterberger M, Pinggera GM, Pallwein L, et al. Plain abdominal radiography with transabdominal native tissue harmonic imaging ultrasonography vs unenhanced computed tomography in renal colic. BJU Int. 2007; 100:887-90.

[11]. Goldwasser B, Cohan RH, Dunnick NR, et al. Role of linear tomography in evaluation of patients with nephrolithiasis. Urology. 1989; 33:253-6.

[12]. Fowler KA, Locken JA, Duchesne JH,et al. US for detecting renal calculi with nonenhanced CT as a reference standard. Radiology.2002; 222:109-13.

[13]. Sheafor DH, Hertzberg BS, Freed KS, et al. Non-enhanced helical CT and US in the emergency evaluation of patients with renal colic: prospective comparison. Radiology. 2000; 217:792-7.

[14]. Ripolles T, Agramunt M, Errando J, et al. Suspected ureteral colic: plain film and sonography vs unenhanced helical CT. A prospective study in 66 patients Eur Radiol. 2004; 14:129-36.

[15]. Ha M, Mac Donald RD. Impact of CT scan in patients with first episode of suspected nephrolithiasis. J Emerg Med. 2004; 27(3):225-31.

[16]. Varanelli MJ, Coll DM, Levine JA, et al. Relationship between during of pain and secondary signs of obstruction of the urinary tract on unenhanced helical CT. AJR Am J Roentgenol. 2001; 177:325-30.

[17]. Sundaram CP, Saltzman B. Urolithiasis associated with protease inhibitors. J Endourol. 1999; 13:309-12. 
[18]. Hermieu JF, Prevot MH, Ravery V, et al. Nephritic colic due to indinavir . Presse Med. 1998; 27:465-67.

[19]. Meagher T, Sukumar VP, Collingwood J, et al. Low dose computed tomography in suspected acute renal colic. Clin Radiol. 2001; 56:873-6.

[20]. Denton ER, Mackenzie A, Greenwell T, Propert R, and Rankin SC. Unenhanced helical CT for renal colic: is the radiation dose justifiable. Clin Radiol. 1999; 54:444-7.

[21]. Mettler FA Jr. Huda W, Yoshizumi TT, et al. Effective doses in radiology and diagnostic nuclear medicine:a catalog. Radiology. $2008 ; 248: 254-63$

\section{ROLE OF NON-CONTRAST CT IN MANAGEMENT OF UROLITHIASIS}

Original Article

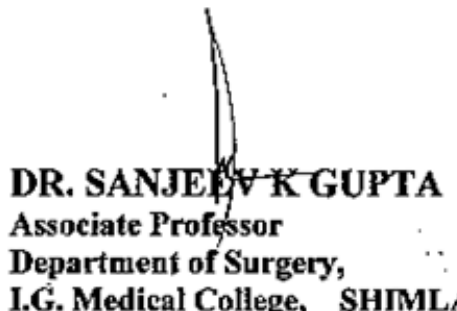

I.G. Medical College, SHIMLA<smiles>C=CC(C)C1(C)CCCC1C</smiles>

DR. AMIT MAINRA

Medical officer

PHC Arki

Distt. Solan.

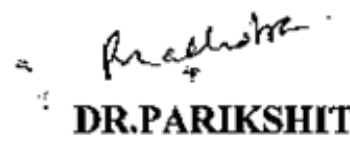

Assistant Professor Department of Surgery, I.G. Medical College, Shimla

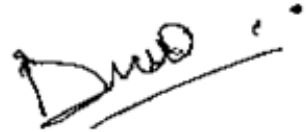

DR. D.K. VERMA, Professor Department of Surgery, I.G. Medical College, SHIMLA 171 001, HP

\section{Address for Correspondence:}

Dr. Sanjcev K Gupta, Highway Apartments, Flat No. 5, Near Petrol Pump, Vikas Nagar, Sbimla-171009 Email: drsnjiv@yahoo.co.in 